\title{
Acquired EGFR C797G Mutation Detected by Liquid Biopsy as Resistance Mechanism After Treatment With Osimertinib: A Case Report
}

\author{
ELISA DE CARLO ${ }^{1}$, MONICA SCHIAPPACASSI ${ }^{2}$, GIACOMO PELIZZARI ${ }^{3}$, TANIA BARESIC ${ }^{4}$, \\ ALESSANDRO DEL CONTE ${ }^{1}$, BRIGIDA STANZIONE ${ }^{1}$, VALENTINA DA ROS ${ }^{1}$, \\ ROBERTO DOLIANA ${ }^{2}$, GUSTAVO BALDASSARRE ${ }^{2}$ and ALESSANDRA BEARZ ${ }^{1}$

\begin{abstract}
${ }^{1}$ Clinical Oncology Department - Centro di Riferimento Oncologico di Aviano (CRO) IRCCS, Aviano, Italy;
${ }^{3}$ Oncology Department, Azienda Sanitaria Universitaria Friuli Centrale (ASUFC), Udine, Italy;

${ }^{4}$ Nuclear Medicine Department - Centro di Riferimento Oncologico di Aviano (CRO) IRCCS, Aviano, Italy
\end{abstract} \\ ${ }^{2}$ Molecular Oncology Department - Centro di Riferimento Oncologico di Aviano (CRO) IRCCS, Aviano, Italy;
}

\begin{abstract}
Background: Osimertinib is a third-generation EGFR-tyrosine kinase inhibitor approved for the treatment of T790M-positive non-small-cell lung cancer. More recently, osimertinib demonstrated improved disease control compared to other EGFR-TKIs. Multiple mechanisms of resistance have been described in T790M-positive patients who experienced treatment failure with osimertinib. Case Report: We report the case of a 78-year-old non-smoker woman with stage IV EGFR L858R-positive lung adenocarcinoma presented with T790M mutation after five years of treatment with gefitinib. The patient was started on osimertinib, but after two and a half years of treatment experienced disease progression. The analyses of circulating tumor DNA using next-generation sequencing showed, together with the pre-existing T790M and exon 21 L858R, the presence of the EGFR C797G resistance mutation. Conclusion: Our case report revealed a rare EGFRdependent acquired resistance mutation to osimertinib in circulating tumor DNA. Liquid biopsy appears to be a promising resource to understand the biology of osimertinib resistance by clonal evolution monitoring and the identification of novel resistance mechanisms.
\end{abstract}

This article is freely accessible online.

Correspondence to: Elisa De Carlo, MD, Medical Oncology Department, Centro di Riferimento Oncologico di Aviano (CRO) IRCCS, via Franco Gallini 2-33081, Aviano, Italy. Tel: +39 (0)0434/659127, e-mail: elisa.decarlo@cro.it

Key Words: Non-small-cell lung cancer, osimertinib, liquid biopsy, EGFR C797G.
Osimertinib is a third-generation EGFR-tyrosine kinase inhibitor (TKI) with proven activity against T790M-positive EGFR mutant non-small-cell lung cancer (NSCLC) patients resistant to first- and second-generation EGFR-TKIs such as gefitinib, erlotinib, and afatinib (1).

More recently, based on the results of the FLAURA phase III trial, osimertinib demonstrated significantly longer progression-free survival and overall survival even when used as first-line treatment, compared to first-generation TKIs (2-3). The osimertinib safety profile was similar to that of the comparator EGFR-TKIs (2-3). However, disease progression eventually occurs during osimertinib therapy and resistance mechanisms may be identified through tissue or liquid biopsy (4). Amplification of MET, HER2, PIK3CA, secondary EGFR mutations (C797S and others), and histologic transformation are the most common resistance mechanisms detected $(5,6)$.

In the current report, we describe the case of a patient with an emerging rare EGFR C797G mutation during osimertinib treatment detected by liquid biopsy. Written informed consent was obtained from the patient for publication of this case report and any accompanying images.

\section{Case Report}

Acquisition of a rare mutation in EGFR Exon 20 (p. C797G, c. $2389 \mathrm{~T}>\mathrm{G}$ ) was detected in a 78-year-old-non-smoker female after the administration of third-generation EGFRTKI osimertinib. In August 2007, the patient underwent left upper lobectomy with diagnosis of EGFR L858R-positive adenocarcinoma, pT2N1M0, followed by adjuvant chemotherapy with four cycles of cisplatin and vinorelbine. The radiological follow-up was performed with positron emission tomography-computed tomography (PET/CT) 

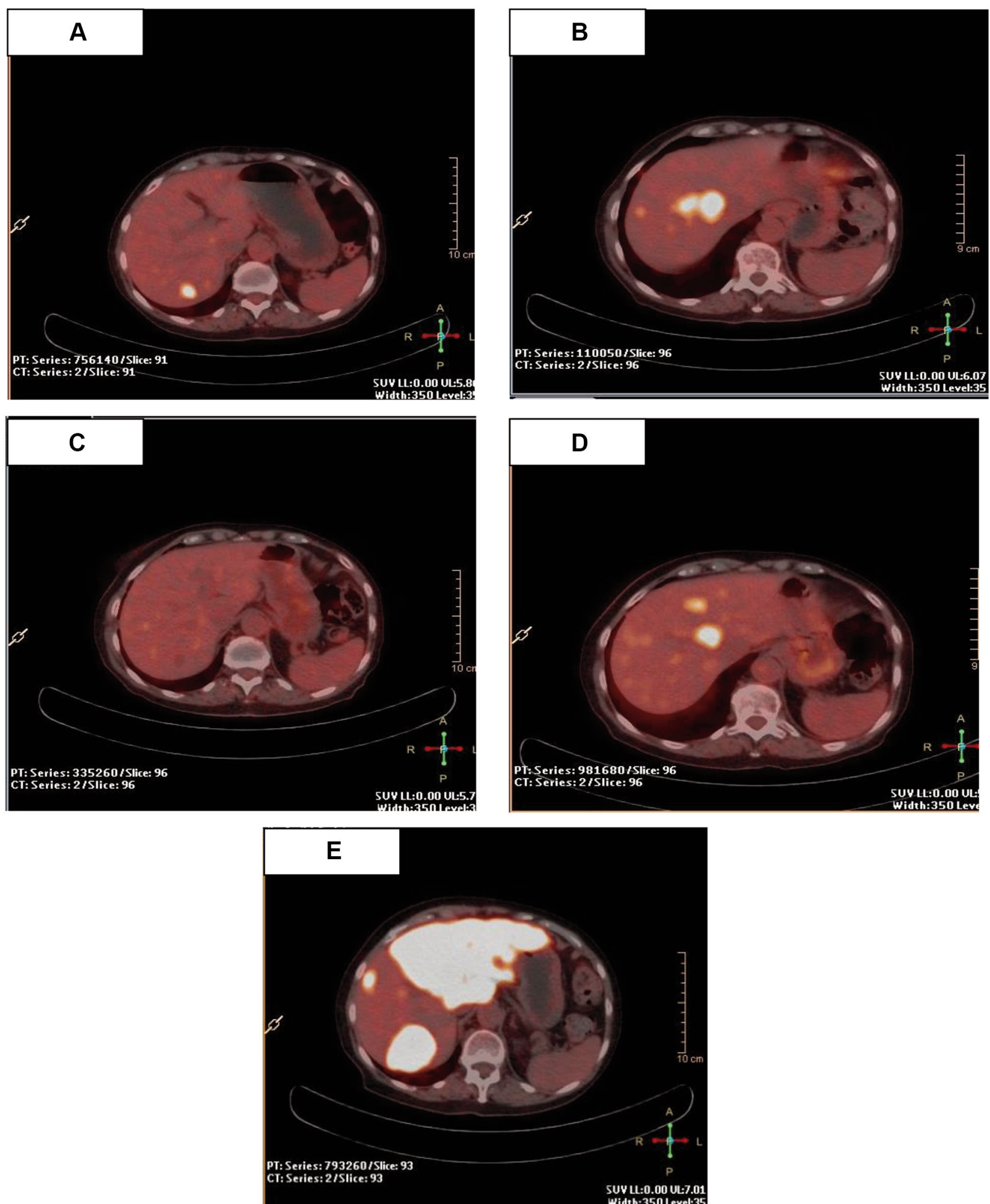

Figure 1. Positron emission tomography scans of liver metastasis at different time points. (A) Liver progression on gefitinib treatment; (B) Liver localizations before osimertinib treatment: a liver biopsy revealed the presence of EGFR exon 21 L858R mutation and the acquisition of T790M mutation; (C) Partial response during osimertinib treatment; (D) Disease progression on osimertinib: a liquid biopsy showed an acquired EGFR C797G mutation together with the pre-existing T790M mutation and exon 21 L858R mutation; (E) Rapidly progressive disease few weeks before death. 
because of hypersensitivity reaction to iodinated contrast medium. In July 2011, the patient presented with cough and dyspnea and a PET/CT demonstrated bilateral lung lesions with mediastinal involvement. Bone scan and brain magnetic resonance imaging (MRI) showed no abnormalities. A bronchoscopy with lung biopsy was performed, with diagnosis of EGFR L858R-positive adenocarcinoma (Exon 21 p.L858R, c.2573T $>$ G). The patient received a first-line treatment with gefitinib, $250 \mathrm{mg}$ per day, with partial remission for five years. In May 2016, a PET/CT revealed new lesions in the liver and right lung (Figure 1). Since the identification of disease progression after first-line gefitinib treatment, the patient was followed-up with liquid biopsy. Subsequent monitoring of circulating tumor DNA (ctDNA) was conducted using amplicon-based ultra deep-nextgeneration sequencing (NGS) on Illumina platform to detect a broader range of mutations.

A liquid biopsy did not reveal a T790M resistance mutation in ctDNA. Unfortunately, it was not possible to perform a tissue confirmatory biopsy because of patient's refusal. Therefore, the patient received chemotherapy with carboplatin and pemetrexed for four cycles, until treatment discontinuation due to nephrotoxicity. In May 2017, the patient experienced shortness of breath and abdominal pain accompanied by radiological progression documented by PET imaging (Figure 1). A subsequent liver biopsy revealed the presence of exon $21 \mathrm{~L} 858 \mathrm{R}$ mutation and the acquisition of Exon 20 T790M mutation (p.T790M, c.2369C>T), also confirmed by liquid biopsy. The patient was then put on osimertinib, $80 \mathrm{mg}$ daily, until PET progression in November 2019 (Figure 1). A liquid biopsy showed an acquired $E G F R$ C797G mutation at a mutant allele frequency (MAF) of $1.3 \%$, together with the pre-existing T790M mutation (MAF: $1.3 \%$ ) and exon 21 L858R mutation (MAF: 1.3\%) (Figure 2). No activating mutations in other oncogene drivers (such as $B R A F, M E T, K R A S$ ) were detected. Unfortunately, the patient never started another treatment due to a rapid general progressive failure that led to her death in February 2020.

\section{Discussion}

Approximately 50-60\% of patients with EGFR-mutant lung cancer treated with first- and second-generation EGFR-TKIs will develop resistance via T790M mutation. Osimertinib is able to overcome this resistance restoring disease control with high response rates and a median progression-free survival of about 10 months, as observed in our clinical case (1). Unfortunately, when resistance to osimertinib occurs without acquired targetable mutations, chemotherapy represents the last therapeutic option for these patients. Common resistance mechanisms to osimertinib involve secondary EGFRdependent mutations, which are found in about one-third of cases (4). The most frequent C797S variant is observed in up

\begin{tabular}{|ccc|}
\hline EGFR & Total reads & MAF $(\%)$ \\
Exon 20 T790M & 34,000 & 1,3 \\
Exon 20 C797G & 34,000 & 1,3 \\
Exon 21 L858R & 25,000 & 1,3 \\
Exon 19 & 45,000 & $(-)$ \\
Exon 18 & 28,000 & $(-)$ \\
\hline
\end{tabular}

Figure 2. Mutant allele frequency (MAF) \% detected on EGFR exons 20 and 21 in the plasma ctDNA by ultra deep NGS. Media coverage of all analyzed amplicons is indicated as Total reads. MAF: Mutant allelic fractions; ctDNA: circulating tumor DNA; NGS: next-generation sequencing.

to $20-25 \%$ of patients, which occurs at the covalent binding site for osimertinib $(7,8)$. Other mutations may occur at G796/C797, L792, or L718/G719 residues (9). Additionally, EGFR-independent pathways may also be activated, with T790M loss and high degree of clonal heterogeneity, mainly involving $M E T, E G F R, K R A S$ amplification and activating mutations in PIK3CA and KRAS (10).

Most data on acquired resistance mechanisms are based on analyses in the second-line setting. Emerging data from both plasma genotyping and from tissue analyses reported differences in frequency of resistance mechanisms to osimertinib given in a front-line versus second-line setting. Patients receiving later-line osimertinib developed more frequently EGFR-dependent resistance compared to patients receiving initial osimertinib (11-13).

Our report describes a rare mutation located at the residue 797 of EGFR, C797G, as a mechanism of acquired resistance to osimertinib. Our patient did maintain the original mutations L858R and T790M, so finally we detected three mutations in the ctDNA. Few reports have identified this mutation from tissue biopsy of NSCLC patients who experienced disease progression on osimertinib, especially in the Asian population (14-16).

A secondary analysis of the AURA3 trial reported the ctDNA genomic profile of T790M-positive NSCLC patients who experienced disease progression during osimertinib treatment. Interestingly, in only one patient among the 73 included in the analysis an EGFR C797G resistance mutation was found by NGS analyzing ctDNA isolated from plasma samples (5).

Our case report underlined the pivotal role of liquid biopsy in establishing the molecular evolution of osimertinib resistance, similarly to other experiences $(17,18)$. Specifically, liquid biopsy, a non-invasive technique, is enabling physicians to monitor targeted treatment response, predicting clinical-radiological progression and the detection of heterogeneous resistance mutations, in order to administer precision-treatment regimens (19-21). Nevertheless, tissue biopsy should be kept into consideration in cases of suspected histologic transformation or non-informative liquid biopsy result. 
Further studies are warranted to investigate the heterogeneity of osimertinib resistance mechanisms, especially using osimertinib as upfront treatment, to guide therapeutic selection to overcome acquired resistance.

\section{Conflicts of Interest}

The Authors declare no conflicts of interest in relation to this study.

\section{Authors' Contributions}

Alessandra Bearz, Elisa De Carlo, Giacomo Pelizzari and Monica Schiappacassi: Conception and preparation of the manuscript, data analysis and interpretation. Tania Baresic, Alessandro Del Conte, Brigida Stanzione and Valentina Da Ros: provision of study materials and data collection. Roberto Doliana and Gustavo Baldassare: provision of study materials, data analysis and interpretation. All Authors contributed and agreed with the content of the manuscript.

\section{Acknowledgements}

This research received no specific grant from any funding agency in the public, commercial, or not-for-profit sectors.

\section{References}

1 Mok TS, Wu Y-L, Ahn M-J, Garassino MC, Kim HR, Ramalingam SS, Shepherd FA, He Y, Akamatsu H, Theelen WS, Lee CK, Sebastian M, Templeton A, Mann H, Marotti M, Ghiorghiu S, Papadimitrakopoulou VA and AURA3 Investigators: Osimertinib or platinum-pemetrexed in EGFR T790M-positive lung cancer. N Engl J Med 376(7): 629-640, 2017. PMID: 27959700. DOI: 10.1056/NEJMoa1612674

2 Soria JC, Ohe Y, Vansteenkiste J, Reungwetwattana T, Chewaskulyong B, Lee KH, Dechaphunkul A, Imamura F, Nogami N, Kurata T, Okamoto I, Zhou C, Cho BC, Cheng Y, Cho EK, Voon PJ, Planchard D, Su WC, Gray JE, Lee SM, Hodge R, Marotti M, Rukazenkov Y, Ramalingam SS and FLAURA Investigators: Osimertinib in untreated EGFR-mutated advanced non-small-cell lung cancer. N Engl J Med 378(2): 113125, 2018. PMID: 29151359. DOI: 10.1056/NEJMoa1713137

3 Ramalingam SS, Vansteenkiste J, Planchard D, Cho BC, Gray JE, Ohe Y, Zhou C, Reungwetwattana T, Cheng Y, Chewaskulyong B, Shah R, Cobo M, Lee KH, Cheema P, Tiseo M, John T, Lin MC, Imamura F, Kurata T, Todd A, Hodge R, Saggese M, Rukazenkov Y, Soria JC and FLAURA Investigators: Overall survival with osimertinib in untreated, EGFR-mutated advanced NSCLC. N Engl J Med 382(1): 41-50, 2020. PMID: 31751012 . DOI: 10.1056/NEJMoa1913662

4 Minari R, Bordi P and Tiseo M: Third-generation epidermal growth factor receptor-tyrosine kinase inhibitors in T790Mpositive non-small cell lung cancer: review on emerged mechanisms of resistance. Transl Lung Cancer Res 5(6): 695708, 2016. PMID: 28149764. DOI: 10.21037/tlcr.2016.12.02

5 Papadimitrakopoulou VA, Han JY, Ahn MJ, Ramalingam SS, Delmonte A, Hsia TC, Laskin J, Kim SW, He Y, Tsai CM, Hida T, Maemondo M, Kato T, Jenkins S, Patel S, Huang X, Laus G,
Markovets A, Thress KS, Wu YL and Mok T: Epidermal growth factor receptor mutation analysis in tissue and plasma from the AURA3 trial: Osimertinib versus platinum-pemetrexed for T790M mutation-positive advanced non-small cell lung cancer. Cancer 126(2): 373-380, 2020. PMID: 31769875. DOI: 10.1002/ cncr.32503

6 Ramalingam S, Cheng Y, Zhou C, Ohe Y, Imamura F, Cho B, Lin M, Majem M, Shah R, Rukazenkov Y, Todd A, Markovets A, Barrett J, Chmielecki J and Gray J: Mechanisms of acquired resistance to first-line osimertinib: Preliminary data from the phase III FLAURA study. Annals of Oncology 29: viii740, 2020. DOI: $10.1093 /$ annonc/mdy424.063

7 Yu HA, Tian SK, Drilon AE, Borsu L, Riely GJ, Arcila ME and Ladanyi M: Acquired resistance of EGFR-mutant lung cancer to a T790M-specific EGFR inhibitor: Emergence of a third mutation (C797S) in the EGFR tyrosine kinase domain. JAMA Oncol 1(7): 982-984, 2015. PMID: 26181354. DOI: 10.1001/ jamaoncol.2015.1066

8 Thress KS, Paweletz CP, Felip E, Cho BC, Stetson D, Dougherty B, Lai Z, Markovets A, Vivancos A, Kuang Y, Ercan D, Matthews SE, Cantarini M, Barrett JC, Jänne PA and Oxnard GR: Acquired EGFR C797S mutation mediates resistance to AZD9291 in non-small cell lung cancer harboring EGFR T790M. Nat Med 21(6): 560-562, 2015. PMID: 25939061. DOI: 10.1038/nm.3854

9 Rotow J and Bivona TG: Understanding and targeting resistance mechanisms in NSCLC. Nat Rev Cancer 17(11): 637-658, 2017. PMID: 29068003. DOI: 10.1038/nrc.2017.84

10 Yang Z, Yang N, Ou Q, Xiang Y, Jiang T, Wu X, Bao H, Tong $\mathrm{X}$, Wang X, Shao YW, Liu Y, Wang Y and Zhou C: Investigating novel resistance mechanisms to third-generation EGFR tyrosine kinase inhibitor osimertinib in non-small cell lung cancer patients. Clin Cancer Res 24(13): 3097-3107, 2018. PMID: 29506987. DOI: $10.1158 / 1078-0432$.CCR-17-2310

11 Schmid S, Li JJN and Leighl NB: Mechanisms of osimertinib resistance and emerging treatment options. Lung Cancer 147: 123-129, 2020. PMID: 32693293. DOI: 10.1016/j.lungcan. 2020.07.014

12 Leonetti A, Sharma S, Minari R, Perego P, Giovannetti E and Tiseo M: Resistance mechanisms to osimertinib in EGFRmutated non-small cell lung cancer. Br J Cancer 121(9): 725737, 2019. PMID: 31564718. DOI: 10.1038/s41416-019-0573-8

13 Schoenfeld AJ and Yu HA: The evolving landscape of resistance to osimertinib. J Thorac Oncol 15(1): 18-21, 2020. PMID: 31864549. DOI: $10.1016 /$ j.jtho.2019.11.005

14 Lee J, Shim JH, Park WY, Kim HK, Sun JM, Lee SH, Ahn JS, Park K and Ahn MJ: Rare mechanism of acquired resistance to osimertinib in Korean patients with EGFR-mutated non-small cell lung cancer. Cancer Res Treat 51(1): 408-412, 2019. PMID: 29807405. DOI: $10.4143 /$ crt.2018.138

15 Nie K, Jiang H, Zhang C, Geng C, Xu X, Zhang L, Zhang H, Zhang Z, Lan K and Ji Y: Mutational profiling of non-small-cell lung cancer resistant to osimertinib using next-generation sequencing in Chinese patients. Biomed Res Int 2018: 9010353 , 2018. PMID: 29713646. DOI: 10.1155/2018/9010353

16 Menon R, Müller J, Schneider P, Lakis S, Thress K, Wolf J, Heukamp L, Heuckmann JM and Griesinger F: A novel EGFR(C797) variant detected in a pleural biopsy specimen from an osimertinib-treated patient using a comprehensive hybrid capture-based next-generation sequencing assay. J Thorac Oncol 
11(9): e105-e107, 2016. PMID: 27086175. DOI: 10.1016/ j.jtho.2016.04.005

17 Ou SI, Cui J, Schrock AB, Goldberg ME, Zhu VW, Albacker L, Stephens PJ, Miller VA and Ali SM: Emergence of novel and dominant acquired EGFR solvent-front mutations at Gly796 (G796S/R) together with C797S/R and L792F/H mutations in one EGFR (L858R/T790M) NSCLC patient who progressed on osimertinib. Lung Cancer 108: 228-231, 2017. PMID: 28625641. DOI: 10.1016/j.lungcan.2017.04.003

18 Zhang Y, He B, Zhou D, Li M and Hu C: Newly emergent acquired EGFR exon 18 G724S mutation after resistance of a T790M specific EGFR inhibitor osimertinib in non-small-cell lung cancer: a case report. Onco Targets Ther 12: 51-56, 2018. PMID: 30588029. DOI: 10.2147/OTT.S188612

19 Rolfo C, Mack PC, Scagliotti GV, Baas P, Barlesi F, Bivona TG, Herbst RS, Mok TS, Peled N, Pirker R, Raez LE, Reck M, Riess JW, Sequist LV, Shepherd FA, Sholl LM, Tan DSW, Wakelee HA, Wistuba II, Wynes MW, Carbone DP, Hirsch FR and Gandara DR: Liquid biopsy for advanced non-small cell lung cancer (NSCLC): A statement paper from the IASLC. J Thorac Oncol 13(9): 1248-1268, 2018. PMID: 29885479. DOI: $10.1016 /$ j.jtho. 2018.05 .030
20 Aggarwal C, Rolfo CD, Oxnard GR, Gray JE, Sholl LM and Gandara DR: Strategies for the successful implementation of plasma-based NSCLC genotyping in clinical practice. Nat Rev Clin Oncol 18(1): 56-62, 2021. PMID: 32918064. DOI: 10.1038/ s41571-020-0423-x

21 Corcoran RB: Liquid biopsy versus tumor biopsy for clinicaltrial recruitment. Nat Med 26(12): 1815-1816, 2020. PMID: 33230339. DOI: 10.1038/s41591-020-01169-6
Received May 22, 2021

Revised June 17, 2021

Accepted June 18, 2021 